\title{
Opposition-Based Differential Evolution for Beta Basis Function Neural Network
}

\author{
Habib Dhahri, Student Member, IEEE, Adel. M. Alimi, Senior Member, IEEE,
}

\begin{abstract}
Many methods for solving optimization problems, whether direct or indirect, rely upon gradient information and therefore may converge to a local optimum. Global optimization methods like Evolutionary algorithms, overcome this problem although these techniques are computationally expensive due to slow nature of the evolutionary process. In this work, a new concept is investigated to accelerate the differential evolution. The opposition-based DE uses the concept of opposite number to create a new population during the learning process to improve the convergence rate of generalization performance of the beta basis function neural network. The proposed algorithm uses the dichotomy research to determine the target solution. Detailed performance comparison of ODE-BBFNN with learning algorithm on benchmarks problems drawn from regression and time series prediction area. The results show that the ODE-BBFNN produces a better generalization performance.
\end{abstract}

\section{INTRODUCTION}

$\mathrm{A}$ rchitecture design of the neural network can be formulated as an optimization problem, where each solution represents architecture. Evolutionary Algorithms (EA) like neural network (NNs) represents an evolving technology inspired by biologically motivated computational paradigms. NNs are derived from the brain theory to simulate the learning behavior of an individual, while EAs are developed from the theory of evolution to evolve whole population toward a better fitness [24], [25]. Although these two technologies seem quite different in the time period action, the number of involved individuals and the process scheme, their similar dynamic behaviors stimulate research on whether a synergistic combination of these two technologies [36] may provide more problem solving power than either alone. There has been extensive analysis of the properties of several classes of neural networks possessing various architectures and training rules. Without the help of a proven guideline, the choice of an optimal network design for a given problem is a difficult process. In this paper, we deal with the so-called Beta Basis Function Neural Network BBFNN [1],[25],[26] that represent an interesting alternative in which we can approximate any function. The beta basis function neural network (BBF) neural network have been widely used in many areas [2], [3], [4], [32] [33] [34]. The Beta basis function has also been used in support vector machines [5] [30]. One of the most important issues in the BBF neural

H. Dhahri is with REGIM: REsearch Group on Intelligent Machines, University of Sfax, National School of Engineers (ENIS), BP 1173, Sfax, 3038, Tunisia, e-mail habib.dhahri@ieee.org.

A.M.Alimi is with REGIM: REsearch Group on Intelligent Machines, University of Sfax, National School of Engineers (ENIS), BP 1173, Sfax, 3038, Tunisia, e-mail adel.alimi@ieee.org. network applications is the network learning, i.e., to optimize the adjustable parameters, which include the centers vectors, the widths of the basis functions and the parameters forms. Another important issue is to determine the network structure or the number of BBF nodes that will be studied in the paper. Interesting aspects of the BBF networks are the possibility of choosing suitable parameters for the hidden units without having to perform a full nonlinear optimization of the network and thus avoiding the risk of getting trapped into poor local minima, and the flexibility to automatically adjust the number of hidden neurons by means of evolution techniques. The computational efficiency of the learning phase comes from the fact that the network has just two layers of weights, and the weights at the output layer are linearly related to the output of the network. There is a large variety of methods that can be combined to produce an adequate BBF network.

The idea of combining DE and NN came up first in the late $90 \mathrm{~s}$, and it had generated an intense field of research in the 1995 s. Since both are autonomous computing methods, why combine them? In short, the problem with neural networks is that a number of parameter has to be set before any training can begin. However, there are no clear rules how to set these parameters. Yet, these parameters determine the success of the training. The differential evolution algorithm is used to find the parameters of a new type of neural network designed by the beta basis function neural network.

The paper provides a set of experimental verification of our proposed approach. In particular, we explore the convergence speed, the effectiveness and the advantage of the new concept over the random ones.

The aim of the proposed paper is to extend the work [6] in which we used the differential evolution algorithm for the design of the beta basis function neural network: this work is also a continuation of the work of Aouiti in [7] [26] that he used the genetic algorithm for design of beta neural system. In spite of the interested results presented in these works, the two algorithms: the differential evolution as the genetic algorithm is very expensive in term of computation time. However, we used a new concept of opposite number in the differential evolution algorithm to improve the convergence time and the performance generalization rate.

This work is organized as follow: in section II, the differential evolution is shortly reviewed. Section III presents the BBF network architecture and discusses some approaches adopted to train this model. One of them, the DE algorithm which presents some related drawbacks and this constitutes the motivation behind ODE development. This is the subject of section IV. The set of experimental results are 
provided in section V. Finally, the work is concluded in section VI.

\section{THE BETA BASIS FUNCTION NEURAL NETWORK (BBFNN)}

In this section, we want to introduce the beta basis function neural network that will be used in the remainder of this paper. The beta basis functions are a special class of functions whose characteristic feature is that their value increases monotonically with the distance from a central point, called center of the BBF.

Besides the centre $c \in I R^{n}$ a beta basis function may also present a width parameter $\sigma \in I R^{n}$, which can be seen as a scale factor for the distance $\|x-c\|$ and the parameter forms $p_{i}$ and $q_{i}$.

The BBF network can be regarded as feed-forward neural network with a single layer of hidden units, whose responses are the outputs of the beta basis functions. The input of each beta basis function of the BBF neural network is the distance between the input vector (activation) and its center.

Fig. 2 shows the schematic diagram of the BBF network with ni inputs, $\mathrm{c}$ beta basis function, and one output units.

If the BBF network consists of $\mathrm{n}$ beta basis function and the outputs units are linear, the response of the ith output units is

$y_{i}=w_{i}^{T} b=\sum_{i=1}^{n} w_{i j} B_{i}\left(x, c_{i}, \sigma_{i}, p_{i}, q_{i}\right), 1 \leq i \leq n_{0}$

$w_{i}=\left[w_{i 0}, w_{i 1}, \ldots, w_{i m}\right]^{T}$ and $\quad b=\left[1, b_{1}, \ldots, b_{m}\right]^{T}$.

Thus the BBF network maps the inputs vector $x \in I R^{n}$ into $\tilde{y}=W b \in I R^{n 0}$,where $\tilde{y}=\left[\tilde{y}_{1}, \tilde{y}_{2}, \ldots, \tilde{y}_{m n}\right]^{T}$ and $\mathrm{W}$ is the weight matrix defined as $W=\left[w_{i 0}, w_{i 1}, \ldots, w_{i m}\right]^{T}$. The adjustable parameters of beta basis function are the centre vector $c=\left[c_{1}, \ldots, c_{m}\right]$, the width vector, $\sigma=\left[\sigma_{1}, \ldots, \sigma_{m}\right]$ the $\quad$ parameters former $p=\left[p_{1}, \ldots, p_{n_{m}}\right], \quad q=\left[q_{1}, \ldots, q{ }{ }_{m}\right]$. The Beta basis function $B_{i}\left(x, c_{i}, \sigma_{i}, p_{i}, q_{i}\right), \mathrm{i}=1, \ldots, \mathrm{n}$, is defined by:

$$
\beta_{i}(x)=\left\{\begin{array}{l}
{\left[1+\frac{\left(p_{i}+q_{i}\right)\left(x-c_{i}\right)}{\sigma_{i} p_{i}}\right]^{p_{i}}\left[1-\frac{\left(p_{i}+q_{i}\right)\left(c_{i}-x\right)}{\sigma_{i} q_{i}}\right]^{q} i} \\
\text { if } x \in]_{x_{0}, x_{1}[} \\
0 \quad \text { else }
\end{array}\right.
$$

where pi $>0$, qi $>0, \mathrm{x}_{0}, \mathrm{x}_{1}$ are the real parameters, $\mathrm{x}_{0}<\mathrm{x}_{1}$ and

$$
c_{i}=\frac{p_{i} x_{1}+q_{i} x_{0}}{p_{i}+q_{i}}
$$

In the multi-dimensional case, the beta function is defined by $\beta(c, \sigma, p, q)(x)=\prod_{i=1}^{i=n} \beta_{i}\left(c^{i}, \sigma^{i}, p^{i}, q^{i}\right)$

The BBF neural network is usually trained to map a vector $x_{k} \in I R^{n} \quad$ in to vector $y_{k} \in I R^{n 0}$ where the pairs $\left(x_{k}, y_{k}\right), 1 \leq k \leq M$ from the training set. If this mapping is viewed as a function in the input space $I R^{n i}$, learning can be seen as function approximation problem .According to this point of view, learning is equivalent to finding the surface in a multidimensional space that provides the best fit to the training data .Generalization is therefore synonymous with interpolation between the data points along the constraint surface generated by the fitting procedure as the optimum approximation to this mapping.

Alimi is the first to investigate the use of the beta basis function in the design of neural network as activation functions (Fig.1) in artificial neural network [1]. Alimi and Hassine [31] proved that BBF networks with one hidden layer are capable of universal approximation. The BBF networks are capable of approximating arbitrarily well any function; also have the best approximation property.

The performance of the BBF network depends to the number of units of beta basis functions, their shapes, the parameters forms, and the method used to determine the associative weight matrix W. Haykin [8] classified The existing learning strategies for neural network as follows:1) learning with a fixed number of units and the centers selected randomly from the training data; 2) supervised learning for the selection of the centers of the network; and 3 ) unsupervised learning for the selection of the fixed number of units[36],[27]. In this paper, we are going to use the second strategies.

One the main problems related to the development of neural network based system is the application of suitable learning algorithm to adjust the network parameters.

Summarizing, the BBF network presents the following adjustable parameters:

Position of BBFs centers $c_{i}$, widths $\sigma_{i}$ of the BBFs, form parameters of the BBFs $p_{i}$ and $q_{i}$, Output weights $w_{i}$.

There are a number of proposals on how to define these parameters in the literature. One first idea is to hold $\mathrm{m}$ fixed and use a gradient descent method to adjust the parameters [9], in a manner very similar to the error back-propagation algorithm, often used with MLPs. Nevertheless, training a BBF network in such a way seems somewhat wasteful, since it does not take advantage of the two-stage training possibility of the BBF architecture. There are several interesting approaches that exploit this potential. Although slightly different, all of them share the same idea: the definition of the hidden layer is considered as the major task [29], since the output weights can be computed according to linear optimization techniques. In $[10,11]$ the authors used the constructive method that allows BBF neural network to 
grow by inserting new units into positions in the feature space where the mapping needs more details.

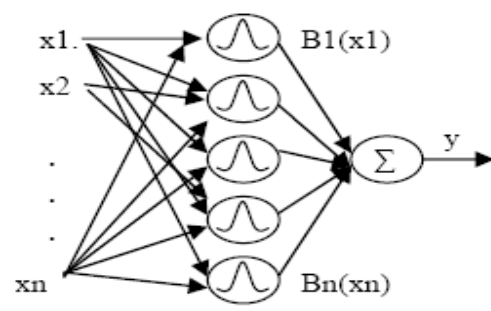

Fig.1.The architecture of BBFNN
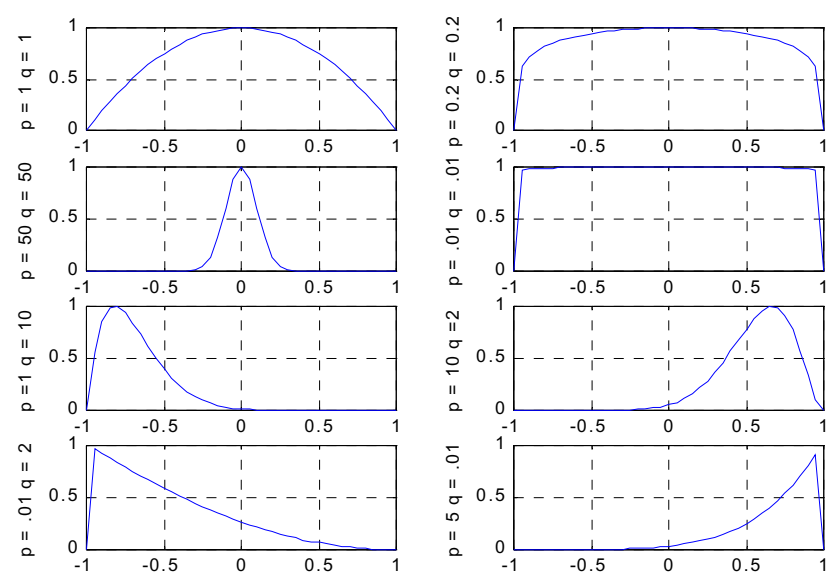

Fig.2.The beta plot in one dimension

\section{THE DIFFERENTIAL EVOLUTION ALGORITHM}

The discovery of differential evolution was dated to the 1995 by Storn and Price [23]. Differential evolution (DE) is a stochastic search algorithm that is originally motivated by the mechanisms of natural selection. Like other evolutionary algorithms, DE is very effective for solving optimization problems with non smooth objective functions, since it does not require derivative information. The DE algorithm was successfully applied in the optimization of some well-known nonlinear, non differentiable and non convex functions by Storn and Price. The DE algorithm is an evolutionary algorithm that uses a rather greedy and less stochastic approach to problem solving than do classical evolutionary algorithms, such as genetic algorithms, evolutionary programming and evolution strategies. In DE, a candidate solution for a specific problem is called an individual or a chromosome and consists of a linear list of genes. Each individual represents a point in the search space, and hence a possible solution to the problem. A population consists of a finite number of individuals. Each individual is decided by an evaluating mechanism to obtain its fitness value. Based on this fitness value and undergoing genetic operators, a new population is generated iteratively with each successive population referred to as a generation. The fitness of an offspring is one-to-one competed with that of the corresponding parent in DE. The potentialities of DE are its simple structure, easy use and local searching property. This drawback could be overcome by employing a larger population. However, by doing so, much more computation time is required to estimate the fitness function.

\section{THE OPPOSITION BASED DIFFERENTIAL EVOLUTION ALGORITHM}

All the technique based to the evolutionary algorithm generates randomly the solutions as an initial population. These candidates' solutions specified by the objective function will be changed to generate new solutions. The best individual in evolved population can be misleading because the applying of the heuristic operators (selection, mutation and crossover) or the update of the position in DE algorithm can steer the population toward the bad solutions. Consequently, the convergence to the guess value is often computationally very expensive. To escape to these drawbacks of the evolutionary algorithms, the dichotomy search is proposed to accelerate the convergence rate and to ameliorate the generalization performance. In other word the search space is halved in to sup-space, in order to decrease the convergence time. Let be $[a, b]$ the search space of the solution $\mathrm{s}$ and $\mathrm{x}$ is the solution belong to the first half. We define a new concept called the opposite number to look for the guess solution in the second half. The ODE algorithm uses the idea of opposite number to create the initial and the evolved populations to improve the performance of DE technique. In the rest of this section, we define the new concept of opposite number in single dimension and multidimension.

Definition: Let $x$ be a real number in the interval $[a, b]$, the opposing number is defined as follows:

$$
\left\{\begin{array}{ll}
\bar{x}=\alpha\left(\frac{a+b}{2}+x\right) \text { if } & x \prec \frac{a+b}{2} \\
\bar{x}=\alpha\left(\frac{a+b}{2}-x\right) \text { if } & x \succ \frac{a+b}{2}
\end{array} \quad \alpha \in[0,1](5)\right.
$$

Definition: Let $M=(x 1, x 2, x 3, \ldots, x n)$ be a point in the $n-$ dimensional space, where $\mathrm{x} 1, \mathrm{x} 2, \mathrm{x} 3, \ldots, \mathrm{xn} \in I R^{n}$ and $\forall i \in\{\mathrm{i} 1,2 \ldots \mathrm{n}\}, \mathrm{xi} \in\left[\mathrm{a}_{\mathrm{i}}, \mathrm{b}_{\mathrm{i}}\right]$. The opposing point of $\mathrm{M}$ is defined by $\bar{M}\left(\bar{x}_{1}, \bar{x}_{2} \ldots, \bar{x} n\right)$ where its components are defined as:

$$
\begin{cases}\bar{x}_{i}=\alpha_{i}\left(\frac{a_{i}+b_{i}}{2}+x_{i}\right) \text { if } & x_{i} \prec \frac{a_{i}+b_{i}}{2}, \alpha_{i} \in[0,1] \\ \bar{x}_{i}=\alpha_{i}\left(\frac{a_{i}+b_{i}}{2}-x_{i}\right) \text { if } & x_{i} \succ \frac{a_{i}+b_{i}}{2}\end{cases}
$$

Let be $[0,1]$ is the interval [a,b], $\mathrm{s}$ is the target solution, $\mathrm{x}$ is the solution proposed by the algorithm (the DE algorithm) and $\bar{x}$ is the opposition of $\mathrm{x}$ so the value of $\bar{x}$ is defined as follows $; \bar{x}=\alpha(0.5-x)$ or $\bar{x}=\alpha(0.5+x)$ according the position of $\mathrm{x}$ to interval center .

Let us consider the two cases in which the potential search space of the original space on one dimension is plotted as a 
line in fig.3. From the illustrated figure, we notice how the concept of the opposite number improves the rate convergence rate of the DE algorithm. We assume in the fist figure, that the estimated solution $\mathrm{x}$ is far from to the target solution $\mathrm{s}$. If the distance $\mathrm{d}$ between the target solution and the estimated by the ODE increase then the distance $\bar{d}$ between the opposite solution and the estimated s decrease. In other word, when the estimated solution is far form the target solution, the opposite solution is near the guess. Now let be treat the case of the solution is near the target solution. According to the second figure, we notice that the opposed target is near to target solution. From the above-stated, the new concept of the opposite- number remains the leader to best solution. That one proves really how the opposite differential evolution ODE enhances the performance convergence of the beta neural system. In the remainder of the section, we explain the proposed algorithm for the training the beta basis function neural network.

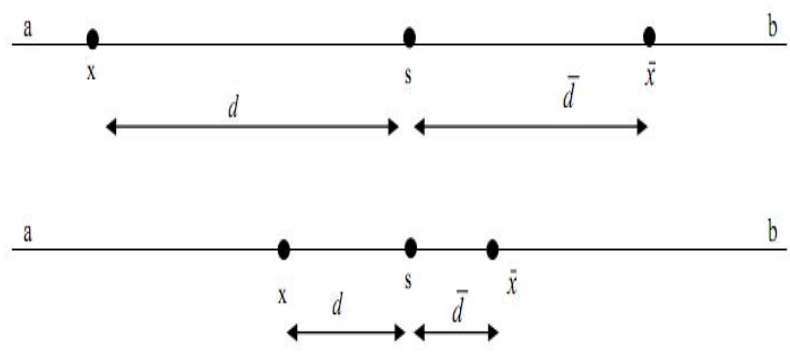

Fig.3. The opposite number

The learning process of ODE is described step by step as follows:

Step 0 (Initialization): At iteration $\mathrm{t}=0$, the initial positions P0ij are generated uniformly distributed randomly;

$$
P 0_{i j}=a_{i j}+\operatorname{rand}_{i}\left(b_{i j}-a_{i j}\right)
$$

Generate the opposite population as follows:

$$
O P 0_{i j} \begin{cases}\alpha_{i}\left(\frac{a_{i}+b_{i}}{2}+P 0_{i j}\right) \text { if } & P 0_{i j} \prec \frac{a_{i}+b_{i}}{2}, \alpha_{i} \in[0,1] \\ \alpha_{i}\left(\frac{a_{i}+b_{i}}{2}-P 0_{i j}\right) \text { if } & P 0_{i j} \succ \frac{a_{i}+b_{i}}{2}\end{cases}
$$

Step1 (individual evaluation): Evaluate the performance of each individual in the population according to the beta neural system. In this paper, the evaluation function $f$ is defined as the error index described in the next section. Select the best from the initial population $\{\mathrm{P} 0, \mathrm{OP} 0\}$

Step 2 (Generate the solution of the next population): At iteration $t$, the vector $X_{r i}$ of the population using the mutation operation. Here, the following mutation operator is adopted

$v_{i}^{d}=x_{r 1}^{d}+F\left(x_{\text {best }}^{d}-x_{r 2}^{d}\right)+F\left(x_{r 3}^{d}-x_{r 4}^{d}\right)$

$o v_{i}^{d}=o x_{r 1}^{d}+F\left(x_{b e s t}^{d}-o x_{r 2}^{d}\right)+F\left(o x_{r 3}^{d}-o x_{r 4}^{d}\right)$
Where $\mathrm{F}$ is real number in $[0,1], o v_{i}^{d}, o x_{r 1}^{d}, o x_{r 2}^{d}, o x_{r 3}^{d}, o x_{r 4}^{d}$ are the opposed vector .

To increase the diversity of the population, each vector changes according to the crossover operator; it defines the following trial vector:

$u_{i}^{d}=\left\{\begin{array}{l}v_{i}^{d} \text { if } \quad \text { rand }(0,1) \leq C r \\ x_{i}^{d} \text { otherwise }\end{array}\right.$

ou $_{i}^{d}=\left\{\begin{array}{l}o v_{i}^{d} \text { if } \quad \text { rand }(0,1) \leq C r \\ \text { ox }{ }_{i}^{d} \text { otherwise }\end{array}\right.$

Where $\mathrm{Cr}$ is the predefined crossover rate constant and the opposed vector are $o v_{i}^{d}, o u_{r 1}^{d}, o x_{i}^{d}$.

Step 3 (select the fittest individual from $\{\mathrm{P}, \mathrm{OP}\}$ ): The ODE learning process ends when a predefined criterion is met. In this paper, the criterion is the goal or total number of iterations.

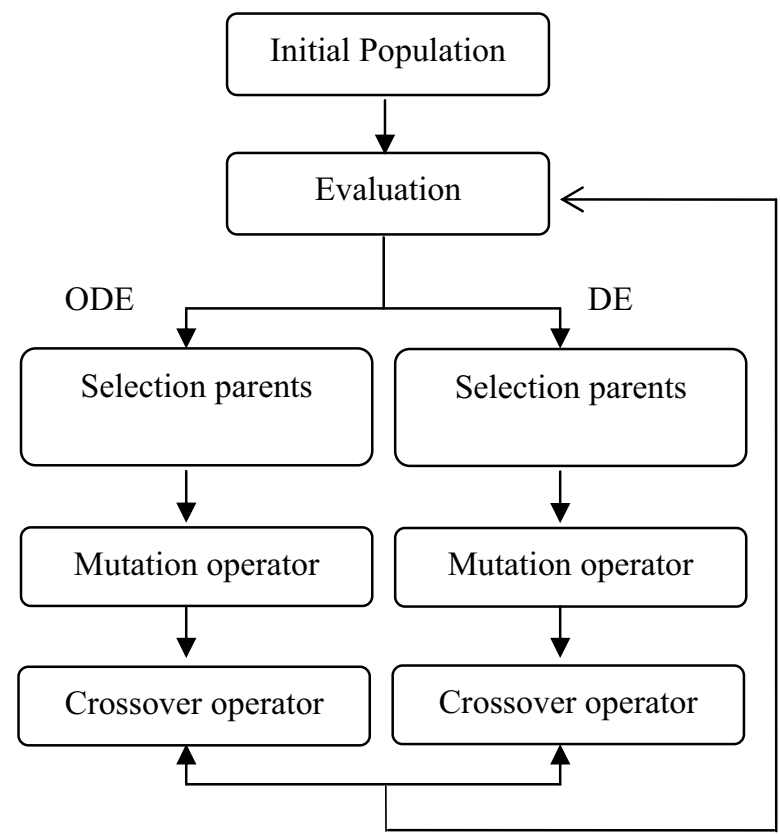

Fig.4.The flowchart of the ODE-BBFNN algorithm

\section{A. The Differential Evolution Encoding}

Like DE, the population size in the ODE employed in this study is equal to NP. Each individual of the population $\mathrm{P}$ correspond to the free parameters in the designed beta neural system. For the beta neural system in Eq. 1 that consists of $\mathrm{n}$ input variables and $\mathrm{m}$ neurons, the chromosome is described by

$$
\begin{array}{ccccccc}
c_{11} & \sigma_{11} & p_{11} & q_{11} \ldots & c_{1 m} & \sigma_{1 m} & p_{1 m} \\
c_{21} & \sigma_{21} & p_{21} & q_{21} \ldots & c_{2 m} & \sigma_{2 m} & p_{2 m} q_{2 m} \\
\vdots & & & \ldots & & & \vdots \\
c_{d 1} & \sigma_{d 1} & p_{d 1} & q_{d 1} \cdots & c_{d m} & \sigma_{d m} & p_{d m} \\
c_{d m}
\end{array}
$$


Tuning BBFs means finding the optimal of the parameters $c_{i}, \sigma_{i}, p_{i}$ and $q_{i}$. These parameters need be adjusted to get the best approximation $\hat{f}$ of the function $\mathrm{f}$. The components $c_{i}, \sigma_{i}, p_{i}, q_{i}, 1 \leq i \leq D$, are codified with the real numbers. These advantages are: No need to make any assumptions about the BBF centers, a better chance for reaching the global optimal solution for the BBF set parameters and ODE-bases approaches can successfully hand non linear equations.

Hence, we use a discrete representation to code the centre $c_{i}$, the width $\sigma_{i}$ and the parameter forms $p_{i}$ and $q_{i}$ of every Beta function. Every four genes that represent a neuron of beta basis function. The number of parameters in the matrix dived by four define the number of neurons in the hidden layer of the network. DE is used to find the global optimal tuning parameters for the ODE-BBFN time series model.

\section{COMPUTIONAL EXPERIMENTS}

The ODE algorithm was tested on various benchmark problems. The comparison of the performance of the proposed algorithm with the literature algorithms .This comparison will mainly focus on the performance of convergence speed, the final approximation error, the generalization error and number of neurons used in the architecture of neural system.

In the first, the ODE algorithm is submitted to time series prediction. This kind of task allows us to evaluate the methods under different conditions.

Particularly, it is possible to generate several training sets. To evaluate its performance, the proposed ODE-BBFN should be submitted to several sets composed of benchmarks functions. To assess the experiments, all the parameters of DE algorithm are adjusted empirically in some cases, intuitively, and do not correspond necessarily to the best possible choice. However, it could also distort the analysis, since a fine tuning is often not possible in real applications.

\section{A. Mackey-Glass time series prediction}

In this sub-section, we describe some applications of the above beta basis function neural network architecture to infer the models associated with some chaotic time series [35]. We consider the Mackey-Glass, one of the most popular and dynamical systems defined as:

$$
\frac{d(x(t))}{d t}=\frac{a x(t-\tau)}{1+x^{c}(t-\tau)}-b x(t)
$$

This time series is chaotic, so there is no clearly defined period. The series will not converge or diverge, and the trajectory is highly sensitive to initial conditions.

The resulting series presents a chaotic behavior and is recognized as a reference problem in the study of neural networks generalization ability. Unfortunately, the exact configuration of the experiment varies from one work to another. Here, the initial values of times series are fixed as follows $a=0.2, b=0.1$ and $\tau=17$. If the $\tau \geq 17$, the time series show the chaotic phenomenon. The goal of prediction Mackey-Glass time series is to predict the $x(t+\Delta t)$ by means $x(t), x(t-\Delta t), \ldots, x(t-n \Delta t)$ as the following equation $x(t+\Delta t)=f(x(t), x(t-\Delta t), \ldots, x(t-n \Delta t)$

where $\Delta \mathrm{t}$ is the sampling interval.

In this paper, the input of neural network BBFNN consists of four data points, $x(t), x(t-6), x(t-12)$ and $x(t-18)$. The output training data corresponds to the trajectory prediction, $x(t+6)=f(x(t), x(t-6), x(t-12), x(t-18))$

For the following experiments, the fourth-order Rung-Kutta method with initial condition $\mathrm{x}(0)=1.2,(x-\tau)=0$ for $0 \leq t \leq \tau$ and the time step 1 was applied to eq. 14 for producing the Mackey-Glass time series data.

One thousand simulation data points are generated from the eq. 14 , the first 500 points are selected as the training data points to build the proposed ODE-BBFNN of Mackey Glass time series, and the second 500 points as the testing points to test the validity of the proposed model.

The learning accuracy measurements are used in all the simulation experiments are the Root Mean Square Error (RMSE) and the MSE and defined as:

$$
R M S E=\sqrt{\frac{\sum_{i=1}^{n}\left(f\left(x_{i}\right)-y_{i}\right)^{2}}{n}}
$$

Where $\bar{y}$ and y denote the mean of the target data and target data, respectively.

In the proposed ODE-BBFNN, the parameters are set as, the population size to 40 , the number of evolving generation to 5000 and the stopping criteria (RMSE) to 0.01and the beta parameters are defined as the center ci and the spread $\sigma \mathrm{i}$ are limited to the upper and lower bound of the input and the form parameters in the interval $[0,5]$.

In order to overcome to the random initialization problem of the parameter, 30 runs generated. Among 30 runs, the root means square (RMSE) of training and testing data obtained using the proposed algorithms are showed in fig. 5 and fig. 6 . Some existing results for this benchmarks problem found in bibliography are also listed for comparison.

After the training, the obtained results are RMSE training and RMSE testing. These results are the best result obtained with the improved ODE-BBFNN method.

Performance comparison results are given in table 1. As observed from this table, ODE-BBFNN achieves the lowest testing and number of nodes as well the training time. 

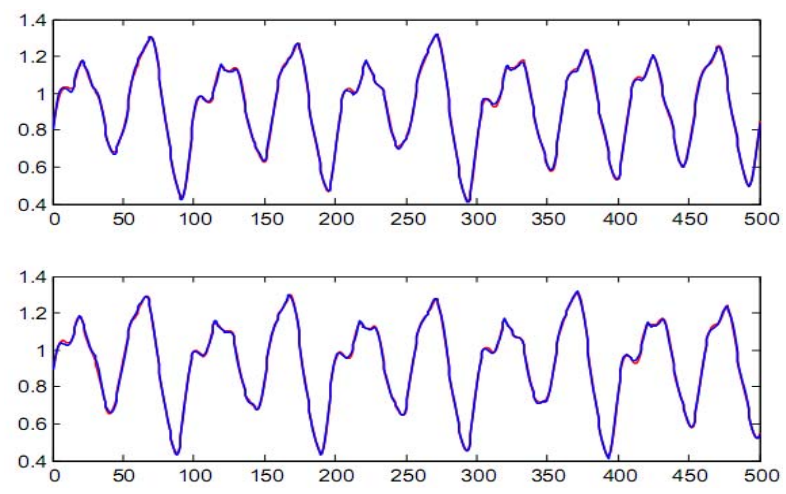

Fig.5. The training and the prediction output

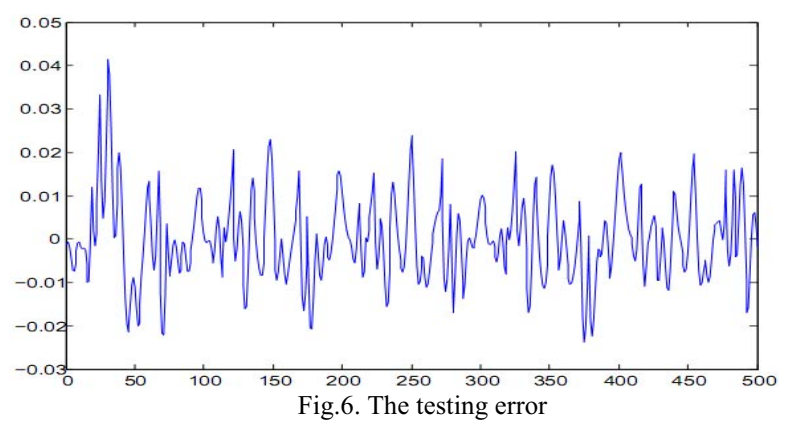

TABLE .1 THE COMPARISON RESULTS OF THE MACKEY -GLASS TIME SERIES

\begin{tabular}{cccl}
\multicolumn{4}{c}{ PREDICTION } \\
\hline Method & $\begin{array}{c}\text { Number of } \\
\text { neurons }\end{array}$ & $\begin{array}{c}\text { RMSE } \\
\text { training }\end{array}$ & $\begin{array}{l}\text { RMSE } \\
\text { testing }\end{array}$ \\
\hline Cho and wang 15] & 23 & 0.0096 & 0.014 \\
Du and Zhang [19] & 98 & 0.0014 & 0.0036 \\
Guang-Bin [16] & 19 & - & 0.031 \\
Yu et al.[21] & 10 & - & 0.009 \\
Harphan and & 116 & - & 0.0015 \\
Dawson [17] & & & \\
Mran [16] & 16 & - & 0.033 \\
Chen et al. [18] & 110 & 0.033 & 0.0036 \\
Gu and Wang [22] & - & - & 0.1095 \\
Yadav et al. [20] & 1 & 0.031 & 0.032 \\
The proposed & 4 & 0.015 & 0.017 \\
method & & & \\
\hline
\end{tabular}

\section{B. Peaks function approximation}

The task was to approximate the following MATLAB Peaks function [12] using the BBFNN:

$$
\begin{aligned}
z=3(1-x)^{2} e^{\left(-x^{2}-(y+1)^{2}\right)}-10\left(\frac{x}{5}-x^{3}-y^{5}\right) e^{\left(-x^{2}-y^{2}\right)} \\
-\frac{1}{3} e^{\left(-(x+1)^{2}-y^{2}\right)}
\end{aligned}
$$

The function mainly consists of a couple of peaks as shown in Fig.8, the training data consisted of 3721 input output data point which were obtained by evaluating the function equ. 18 over $61 * 61$ uniformly distributed grid on the square $[-3,3] \times[-3,3]$ in $x-y$ plane. Three hidden neurons were used. The learning rate was state to 0.0001 . The comparison of the proposed algorithm is mated with the performance of the PIL algorithm and the standard online BP algorithm. Thirteen numerical simulations were used to escape to initialization problem of the parameters. The table 2 shows the MSE values of these algorithms. By comparing the results presented in the table 2, we remark that ODEBBFNN algorithm "won the competition" in terms of error rate, in terms of number of neurons and also in terms of time for the ten runs. The proposed algorithm achieves the better solution in a few numbers of epochs. So the ODE gives the better solution convergence speed than the PIL and online BP learning algorithm The Fig.7 shows the error training.

TABLE..2. MSE $\left(\times 10^{2}\right)$ OF THE TRAINED NEURAL NETWORK

\begin{tabular}{|c|c|c|c|c|c|c|c|c|c|c|}
\hline & 1 & 2 & 3 & 4 & 5 & 6 & 7 & 8 & 9 & 10 \\
\hline PIL & 0.29 & 0.24 & 0.31 & 0.29 & 0.34 & 0.37 & 0.29 & 0.44 & 0.24 & 0.34 \\
\hline BP & 0.39 & 0.72 & 0.28 & 0.23 & 0.52 & 0.23 & 0.32 & 0.72 & 0.72 & 0.32 \\
\hline SDLM & 0.50 & 0.63 & 0.24 & 0.51 & 0.50 & 0.45 & 0.45 & 0.75 & 0.35 & 0.75 \\
\hline $\begin{array}{c}\text { Training MSE } \\
\text { ODE }\left(\times 10^{4}\right)\end{array}$ & 0.19 & 0.23 & 0.17 & 0.16 & 0.16 & 0.15 & 0.14 & 0.20 & 0.18 & 0.17 \\
\hline $\begin{array}{c}\text { Testing MSE } \\
\text { ODE }\left(\times 10^{4}\right)\end{array}$ & 0.2 & 0.23 & 0.16 & 0.16 & 0.18 & 0.16 & 0.15 & 0.21 & 0.21 & 0.20 \\
\hline
\end{tabular}

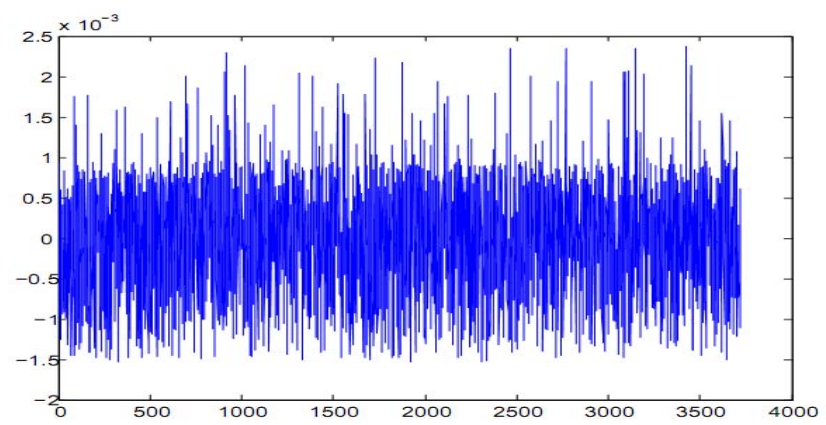

Fig.7. The training error of Peak function approximation

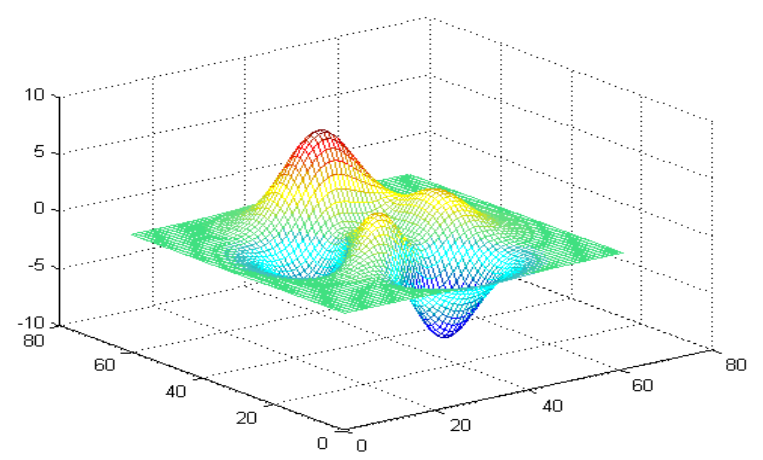

Fig.8. Peaks function 


\section{Gabor function approximation}

Now, we use the ODE-BBFNN to approximate the 2-D Gabor function [12], shown in fig. 9:

$$
z=\frac{2}{\pi} e^{\left(-2\left(x^{2}+y^{2}\right)\right.} \sin (2 \pi(x+y))
$$

The training data are uniformly distributed on the square $[-1.5,1.5] \times[-1.5,1.5]$ in $\mathrm{x}-\mathrm{y}$ plane. Three hidden neurons were used for the training the beta basis function neural networks whereas the three algorithms (PIL, SDLM, BP) used ten hidden neurons for approximate this function. As the first simulation, we repeat the simulation for 30 runs to escape of the random initialization of the population and the opposed population. The proposed algorithm achieve the desired MSE in a number of training epochs less than 500 epochs, whereas ,the three algorithms reach the steady -state MSE in 50000 iterations in all the ten runs. In this simulation study, the Gabor function approximation was shown to be a "simple problem" for the ODE-BBFNN for all the runs, whereas this function was shown as a "hard problem" for the cited algorithm. Fig.10 shows a typical error learning process. Table 3 shows the MSE values of the three algorithm trained by PIL, SDLM and ODE algorithms for all the runs. It seen from the table that ODE algorithm "won the competition" for all the ten runs in terms of convergence speed, in terms of training error and in terms of number of hidden units.

TABLE 3 .MSE $\left(\times 10^{2}\right)$ OF THE TESTING NETWORK

\begin{tabular}{|c|c|c|c|c|c|c|c|c|c|c|}
\hline & 1 & 2 & 3 & 4 & 5 & 6 & 7 & 8 & 9 & 10 \\
\hline PIL & 0.26 & 1.72 & 0.47 & 1.72 & 0.22 & 0.40 & 0.22 & 0.37 & 0.18 & 0.18 \\
\hline SDLM & 0.86 & 0.18 & 0.89 & 0.8 & 0.26 & 0.12 & 0.86 & 0.87 & 0.32 & 0.38 \\
\hline $\begin{array}{c}\text { Training MSE } \\
\text { ODE }\left(\times 10^{3}\right)\end{array}$ & 1.4 & 1.3 & 1.46 & 1.41 & 1.1 & 1.56 & 1.43 & 1.6 & 1.35 & 1.1 \\
\hline $\begin{array}{c}\text { Testing MSE } \\
\text { ODE }\left(\times 10^{3}\right)\end{array}$ & 1.38 & 1.5 & 1.37 & 1.55 & 1.3 & 1.7 & 1.25 & 1.5 & 1.46 & 1.4 \\
\hline
\end{tabular}

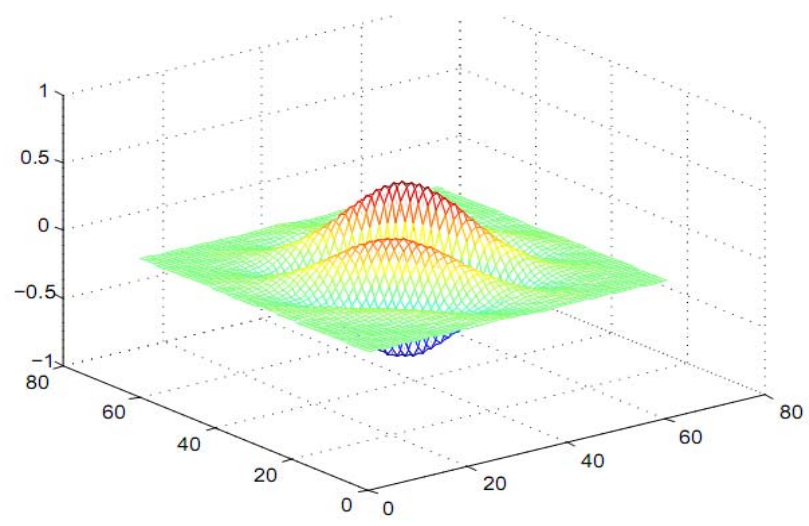

Fig.9. Gabor function

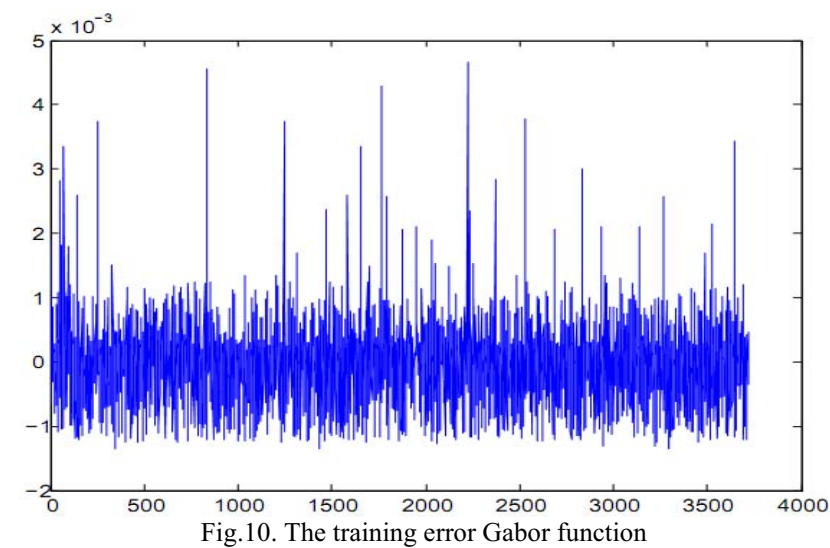

\section{CONCLUSION}

A new concept for the differential evolution algorithm, called opposition based differential evolution has been presented. The essence of the ODE approach is that to accelerate the convergence speed to the best solution. Since the $\mathrm{DE}$ algorithm is very expensive, the new concept of opposite number improve the computation time of the algorithm. The sharing of the input space in two sub-spaces improves the process of the determination of the optimal solutions in short time in comparing it to classic differential evolution.

The following conclusion can be drawn from the benchmark studies and analysis.

The ODE is compared for different problem dimension; the results confirm that ODE performs better over in the simulation problems. For these kinds of problems, a large number of nodes are required. On the other hand, ODE performs well with a fewer number of nodes.

Compared to three algorithms: PIL,BP and SDLM, the proposed algorithm have the high solution for all the runs in terms of convergence speed, number of training error and also in terms of number units used in the hidden layers.

\section{ACKNOWLEDGMENT}

The authors would like to acknowledge the financial support of this work by grants from General Direction of Scientific Research (DGRST), Tunisia, under the ARUB program.

\section{REFERENCES}

[1] A.M. Alimi, "The Beta System: Toward a Change in Our Use of Neuro-Fuzzy Systems," International Journal of Management, Invited Paper, June, pp. 15-19, 2000.

[2] H. Dhahri, A. M. Alimi, "Automatic Selection for the Beta Basis Function Neural Networks," Nature Inspired Cooperative Strategies for Optimization (NICSO), pp. 461-474, 2007.

[3] R. Hassine, F. Karray, A.M Alimi et al."Approximation properties of fuzzy systems for smooth functions and their first-order derivative," IEEE Transactions on Systems, Man, and Cybernetics Part A: Systems and Humans, vol. 33 (2), pp.160-168, 2003.

[4] C. Aouiti, A.M. Alimi, and A. Maalej, "A Genetic Designed Beta Basis Function Neural Network for Approximating Multi-Variables 
Functions," in V. Kurkova et al. (eds.) Artificial Neural Nets and Genetic Algorithmes, Springer-Verlag, Wien, pp. 383-386 ,2001

[5] M.T. Hamdani, and A.M. Alimi, "How are beta_SVM good kernels, "in Proc. World Computer Congress: WCC'2004, Toulouse, France, 2004, pp. 283-292.

[6] H. Dhahri, A.M. Alimi, F. Karray," The modified particle swarm optimization for the design of the Beta Basis Function neura networks," In Proc. Congress on Evolutionary Computation, Hong Kong, China, 2008, pp. 3874-3880. 2008.

[7] C. Aouti, A.M. Alimi, F. Karray, A .Maalej," The design of beta basis function neural network and beta fuzzy systems by a hierarchical. Fuzzy Sets Syst. 154:251-274, 2005.

[8] H. Simon, '’Neural networks: a comprehensive foundation', New Jersey, Printice Hall, 1994.

[9] H. Dhahri and A.M. Alimi, "Hierarchical Learning Algorithm for the Beta Basis Function Neural Network," in Proc. Third International Conference on Systems, Signals \& Devices, Tunisia, 2005.

[10] M. Njah, A.M. Alimi, M. Chtourou and R. Tourki , "Algorithm of Maximal Descent AMD for training Radial Basis Function Neural Networks ," in Proc. IEEE International Conference on Systems, Man and Cybernetics: SMC'02, Hammamet, Tunisia, October 2002.

[11] C. Aouiti, A.M. Alimi, Karray F, Maalej A, "A hierarchical genetic algorithm for the design of beta basis function neura network," In Proc. of the International Joint Conference on Neural Networks, 2002, pp.1246 - 1251

[12] S. Wang and L.E. Banta, "Parameter Incremental Learning Algorithm for Neural network," IEEE Trans .Neural network, vol.17, pp.14241438, 2006.

[13] M.C. Mackey, L. Glass, "Oscillation and chaos in physiological control systems", Science, vol.197, pp. 287-289, 1977.

[14] H. Dhahri, A.M. Alimi, F. Karray: "The modified particle swarm optimization for the design of the Beta Basis Function neural networks," in proc. IEEE Congress on Evolutionary Computation, 2008, pp. 3874-3880.

[15] K.B. Cho, B.H. Wang, Radial basis function based adaptive fuzzy systems and their applications to system identification and prediction, "Fuzzy Sets Syst. Vol. 83 ,1996, pp. 325-339.

[16] H. Guang-Bin, P. Saratchandran, N. Sundararajan,"A generalized growing and pruning RBF (GGAP-RBF) neural network for function approximation”. Trans. on Neural Networks, vol.16, no.1, pp. 57- 67, 2005.

[17] C. Harpham, C.W. Dawson, "The effect of different basis functions on a radial basis function network for time series prediction: a comparative study," Neurocomputing vol.16, pp.2161-2170, 2005.

[18] Y. Chen, B. Yang, J. Dong, "Time-series prediction using a local linear wavelet neural network", Neurocomputing, vol. 69, pp. 449 465, 2006.

[19] H. Dua, N. Zhanga, "Time series prediction using evolving radial basis function networks with new encoding scheme," Neurocomputing ,vol.71, pp.1388-1400,2002.

[20] R.N. Yadav , P.K. Kalra, J. John, "Time series prediction with single multiplicative neuron model". Applied Soft Computing, vol. 7, pp.1157-1163, 2002.

[21] Y.Yi Fu, C. Wu, J.g Jeng and C. Ko , "ARFNNs with SVR for prediction of chaotic time series with outliers," Expert Systems with Applications, 2009. (Accepted for publication),

[22] H. Gu and H. Wang, "Fuzzy prediction of chaotic time series based on singular value decomposition," Applied Mathematics and Computation, vol.185, pp. 1171-1185, 2007.

[23] R. storn, "Differential evolution -a simple and efficient adaptive scheme for global optimization over continuous space," J. global optim. vol.4, pp.341-359, 1995.

[24] I. Kallel, M. Jmaiel, A.M. Alimi, "A multi-agent approach for genetic algorithm implementation", in Proc. of the IEEE International Conference on Systems, Man and Cybernetics, vol.7, 2002, pp. 358363.

[25] M. Masmoudi, M. Samet, A.M. Alimi, "A bipolar implementation of the Beta neuron," Inter. Journal of Electronics, vol. 87, no. 6, pp. 675 682, 2000.

[26] C. Aouiti, A.M Alimi, F. Karray A. Maalej, "A hierarchical genetic algorithm for the design of beta basis function neural network", in
Proc. of the International Joint Conference on Neural Networks, vol.2, 2002, pp. 1246-1251.

[27] H. A. Zouari, L.A Heutte, Y.A. Lecourtier ,A.M. Alimi, "Building diverse classifier outputs to evaluate the behavior of combination methods: The case of two classifiers," Lecture Notes in Computer Science (including subseries Lecture Notes in Artificial Intelligence and Lecture Notes in Bioinformatics), vol. 3077, 2004, pp. 273-282 .

[28] I. Kallel, N. Baklouti, A.M. Alimi, "Accuracy preserving interpretability with hybrid hierarchical genetic fuzzy modeling: Case of motion planning robot controller, "in Proc. of the International Symposium on Evolving Fuzzy Systems, Lake District, UK, Sept. 2006, pp. 312-317.

[29] H. Bezine, N. Derbel, A.M. Alimi, "Fuzzy control of robot manipulators: Some issues on design and rule base size reduction," Eng. Applications of Artificial Intelligence, vol. 15, no.5, pp. 401-416, Sep. 2002.

[30] M.T. Hamdani, M.A. Alimi, and F. Karray, "Distributed Genetic Algorithm with Bi-Coded Chromosomes and a New Evaluation Function for Features Selection, " in Proc. IEEE Congress on Evolutionary Computation, Vancouver, CA , 2006, pp. 2596-2603.

[31] R. Hassine, F. Karray, A.M. Alimi, M. Selmi, "Approximation properties of piece-wise parabolic functions fuzzy logic systems, "Fuzzy Sets and Systems, vol.157, no.4, pp. 501-515, 2006.

[32] A.M. Alimi, "Evolutionary neuro-fuzzy approach to recognize on-line Arabic handwriting, "in proc. of the International Conference on Document Analysis and Recognition, ICDAR, vol.1, 1997, pp. 382386.

[33] A.M. Alimi, "Neuro-fuzzy approach to recognize Arabic hand written characters, "in Proc. of the IEEE International Conference on Neural Networks, ICNN, vol. 3, 1997, pp. 1397-1400.

[34] A.M. Alimi, "Evolutionary computation for the recognition of on-line cursive handwriting, "IETE Journal of Research, vol.48, no.5, pp. 385-396, SPEC., Sep. 2002.

[35] A.M. Alimi, N. Derbel, "New hierarchical control algorithm for large scale time-delay systems, " Control and computers, vol. 23, no.2, pp. 48-52, 1995

[36] H. A. Zouari, L.A Heutte, Y.A. Lecourtier ,A.M. Alimi, " Simulating classifier outputs for evaluating parallel combination methods, "Lecture Notes in Computer Science (including subseries Lecture Notes in Artificial Intelligence and Lecture Notes in Bioinformatics), vol. 2709, 2003, pp 296-305. 